\title{
160-Gbit/s Packet Clock Distribution with Instantaneous Synchronization and Low Timing Jitter
}

Fausto Gomez-Agis, Nicola Calabretta, Aaron Albores-Mejia, Oded Raz, and Harm J. S. Dorren

COBRA Research Institute, Eindhoven University of Technology, P.O. Box 513, NL-5600 MB, Eindhoven, The Netherlands, $\bowtie$ f.gomez-agis@tue.nl

Abstract A novel packet clock distribution concept based on insertion of in-band clock pilot is presented. Experimental results for 160 Gbit/s OTDM packet data indicate a low timing jitter of 250 fs for the bursty clock enabling error-free operation with $1 \mathrm{~dB}$ penalty.

\section{Introduction}

Optical packet switching (OPS) is visualised as a solution to efficiently achieve maximum bandwidth utilization and reducing traffic bottlenecks in optical networks ${ }^{1}$. So stringent requirements on the processing of individual packets in switching nodes becomes fundamental. This implies that tasks such as optical routing and clock recovery must be performed on a packet-by-packet basis. Typical clock recovery techniques based on electroabsorption modulators, ring lasers or phase-locked loops require long acquisition and lock-in times being more suitable for continuous serial data than for data packets. Clock recovery in an asynchronous mode must provide a bursty clock with the same duration of the incoming data packet. So clock recovery methods providing fast-locking times, low timing jitter, low persistence time and stability are of great interest. In this paper we present a new packet-based clockdistribution concept. Extending the ideas presented in ${ }^{2}$, we demonstrate clock distribution for $160 \mathrm{Gbit} / \mathrm{s}$ OTDM packets. The concept is based on the insertion of a clock pilot which is packetized together with the data at the transmitter and extracting this pilot signal at the receiver. The extracted pilot is the asynchronous or bursty clock employed to drive a two-stage electroabsorption (EAM)-based demultiplexer achieving error-free operation from 160 to-10 Gbit/s. This method provides instantaneous and self-synchronization, high stability and low timing jitter.

\section{Experimental setup}

The experimental setup that allows for fast asynchronous clock distribution of packet-based data is schematically shown in Fig. 1. The system consist of two main blocks: the transmitter and the receiver. In the transmitter, optical pulses generated by a mode-locked fiber ring laser (MLFRL) at $1549.8 \mathrm{~nm}$ with duration of $1.4 \mathrm{ps}$ and $40 \mathrm{GHz}$ repetition rate, are encoded with a user pattern from a $40 \mathrm{Gbit} / \mathrm{s}$ pulse pattern generator (PPG1) to form the optical data sequence. The limited user pattern of the PPG resulted in a 64-bit payload based on a $2^{7}-1$ PRBS with a total duration of $6.4 \mathrm{~ns}$. The encoded pulses are sent through a fiber interleaver and are time-multiplexed to constitute the $160 \mathrm{Gbit} / \mathrm{s}$ bit-stream. To accomplish asynchronous clock distribution in the receiver, we employed the in-band self-clock distribution method $^{2}$. The signal is coupled into an optical fiber bragg grating (FBG1) with $0.4 \mathrm{~nm}$ bandwidth and $30 \mathrm{~dB}$ supression of the rejection band that carves a portion of the spectrum where a pilot signal is inserted. The central wavelength of the FBG1 is centered at $1552.52 \mathrm{~nm}$. The pilot is generated by modulating a CW laser $\left(\lambda_{p}=1552.52\right.$ $\mathrm{nm}$ ) with a master clock at $10 \mathrm{GHz}$ and combined with the signal through an optical coupler. Subsequently, to form the packets and the asynchronous clock, the signal together with the pilot are coupled into an intensity modulator driven by PPG2 which encodes the envelope of the packets.

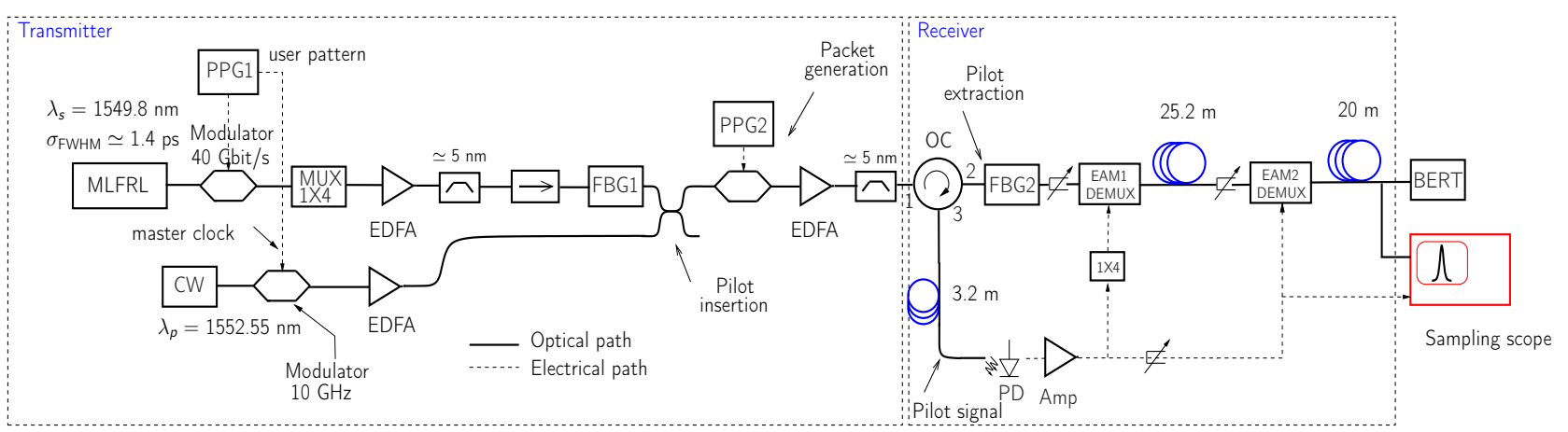

Fig. 1: Packet-based clock distribution concept: experimental setup. FBG: fiber bragg grating; PPG: pulse pattern generator; OC: optical circulator; EAM: electro-absorption modulator. 


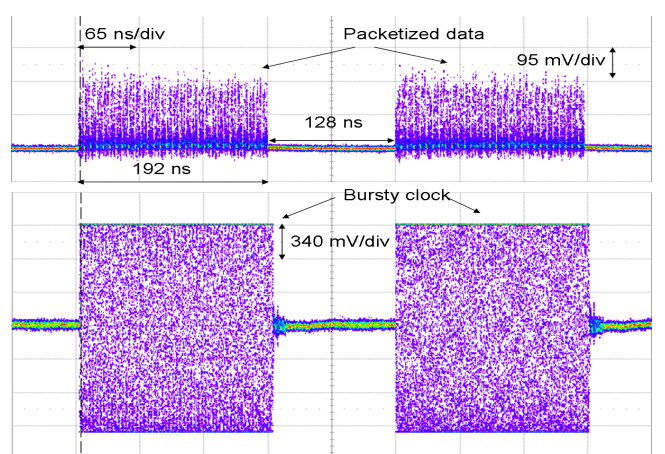

Fig. 2: Time domain traces of the packetized data and bursty clock.

The periodicity of the packets is of 320 ns where the "ones" has 192 ns duration corresponding to 30 times the data payload $(30 \times 6.4 \mathrm{~ns})$. The data density of the payload is $90 \%$. The "zeroes" occupy the remaining $10 \%$ in order to serve as a guard-band within the boundaries of the generated envelope, which holds a rise and falling time within the $10-90 \%$ of 250 ps. As the pilot and the data are synchronized in the transmitter and are spectrally located close to each other, they are affected by the same impairments leading to the same phase drifts. Therefore, the relative phase between the clock and the data is preserved. If the data and the clock are time-aligned in a demultiplexer, the channel extraction can be achieved. In the receiver, the packetized data and the pilot are separated via an optical circulator and FBG2. FBG2 holds a central frequency and bandwidth identical to FBG1. Time domain traces of the packetized data and bursty clock are shown in Fig. 2. The extracted pilot is converted to the electrical domain by a photoreceiver whose output is used to drive a two-stage electroabsorption (EAM)-based demultiplexer. The first EAM is operated at $40 \mathrm{GHz}$ and the cascaded EAM at $10 \mathrm{GHz}$. The received clock is split, amplified and correspondingly quadrupled $(\times 4)$ to satisfy the operating conditions of the demultiplexer. The spectrum of the data after pilot extraction in port 2 is shown in Fig. 3. Notice the carved spectrum. The inset waveform indicates a clear eye after pilot extraction. Finally, the performance of the demultiplexed data is evaluated in a bit-errorrate tester (BERT) and a sampling scope.

\section{Experimental Results}

First, phase noise measurements were performed on the extracted bursty clock in the range between $100 \mathrm{~Hz}$ to $100 \mathrm{MHz}$. As shown in Fig.4, the peaks between the frequency offset of $6 \mathrm{MHz}$ and $100 \mathrm{MHz}$ corresponds to the $N^{\text {th }}$ harmonic associated to the fundamental frequency of the envelope of the packets (320 ns $\rightarrow 3.125 \mathrm{MHz}$ ).

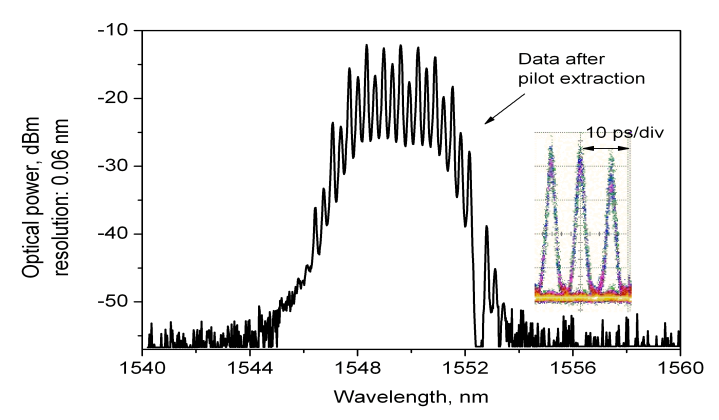

Fig. 3: Optical spectrum of the packetized data after pilot extraction. Inset: eye-diagram.

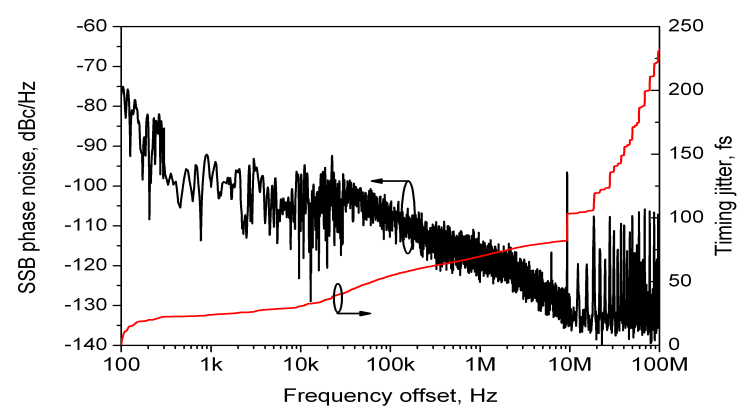

Fig. 4: Single-sideband phase noise and timing jitter of the distributed bursty clock.

Despite these peaks, the integration of the noise spectrum reveals a timing jitter of $250 \mathrm{fs}$. This value corresponds to the operational characteristics required to achieve error-free at $160 \mathrm{Gbit} / \mathrm{s}^{3}$.

Next, we investigate the performance of the proposed concept. The data after pilot extraction is fed to the two-stage EAM-based demultiplexer driven by the distributed electrical bursty clock and the evaluation of the extracted data is performed by a BER tester. In order to demultiplex correctly, the synchronization between the packets and the bursty clock has to be taken into account. Namely, at every demultiplexing stage the packets and the bursty clock must have the same arrival time, since any time-misaligment will produce a distorted output and error-free operation will not be achieved. The difference in the path length between the electrical clock and the optical data at each stage of the demultiplexer requires compensation within picosecond accuracy. This is achieved by manipulating the delay between the data and the bursty clock to optimise the alignment. This alignment is also critical for qualitative evaluation at the sampling scope to trigger the acquisition at the very moment of the arrival of the data.

The BER evaluation of the best and worst demultiplexed channels are shown in Fig. 5. The black lines represent BER measurements of the 


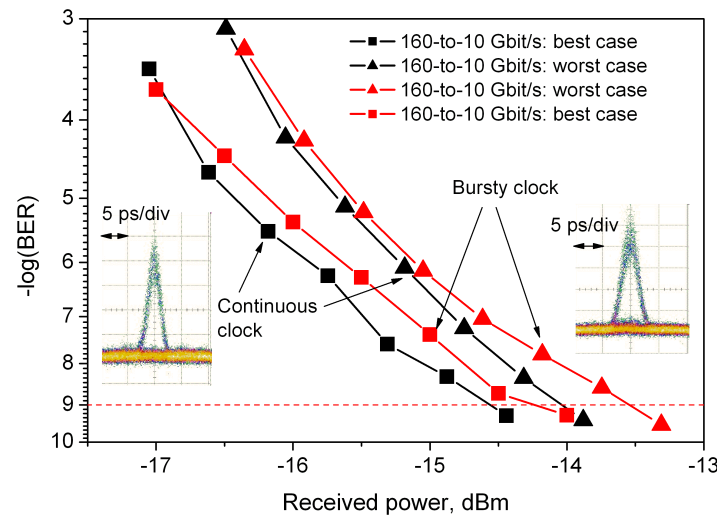

Fig. 5: BER curves of the $10 \mathrm{Gbit} / \mathrm{s}$ channels: using the master clock (black lines) and the bursty clock (red lines) to drive the EAM-based demultiplexer. Squares: best case; triangles: worst case.

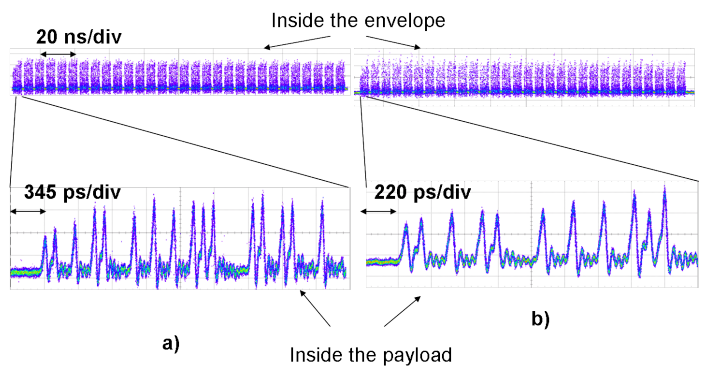

Fig. 6: Qualitative evidence of the packet-based clock distribution concept. Data content inside the envelope and inside the payload visualised in a sampling scope employing as a trigger the recovered bursty clock (a) and a continuous word-frame clock (b).

extracted channels employing a continuous clock to drive the demultiplexer where the square and triangle symbols show the performance of best and worst channels respectively as a reference. In this scenario, the alignment between the data and clock in the demultiplexer is not critical to the extraction of the packet due to the continuity of the clock. The red lines represent the BER evaluation of the extracted channels using the distributed bursty clock to drive the demultiplexer. The square and triangle show the performance of the best and the worst channel in this case. The insets show a clear eye diagrams of the extracted pulses.

Error-free operation is achieved within $1 \mathrm{~dB}$ penalty. This penalty can be attributed to some shaping effect of the envelope on the packets and to the demultiplexing window produced by some missalignment between the packets and the bursty clock. The difference between the best and worst case can be due to a not well equalized channel. Due to the limitation in the available BERT, a bursty clock can not be used to trigger the error evaluation, instead we supply the master clock in order to keep running the BERT continuously. Also, we provide an additional evidence of the packet-based clock distribution method operation by means of a qualitative test, which consists on observing the data inside the packet and the content inside the payload. The test was performed in a sampling scope in two modes. In the first mode we provide the reference wordframe clock coming from PPG2, which was used as a trigger, while in the second one the bursty clock was provided. In the first mode of operation no alignment is required due to the continuity of the clock whereas in the second mode the timealignment between the packetized data and the bursty clock must be satisfied, otherwise no signal can not be visualised. In Fig. 6 can be observed snapshots of the data content inside the envelope and inside the payload acquired by the sampling scope for both modes. In the image, 30 payloads can be recognized inside the envelope, as well as, the individual bits inside the payload. Due to the limitation in the available scope, a bursty clock can not be used to visualise the eye-diagram.

\section{Conclusions}

This paper reports on the operation of a 160 Gbit/s OTDM data packets clock distribution concept. The method provides instantaneous synchronization, highly stable performance and error-free operation for time-demultiplexing tributaries at $10 \mathrm{Gbit} / \mathrm{s}$. Two comments have to be made. Firstly, in the experiments packets are separated by a guard-band of 128 ns. The duration of the guard band simplified the time alignment of packetized clock and data at the demultiplexer, but fundamentally the guard band is limited only by the switching of the packet generator switch. Secondly, this clock distribution experiment did not include a transmission link. This is because the BER tester cannot be gated with a bursty clock. In $^{2}$, continuous in-band clock distribution experiments have been carried out over a $50 \mathrm{~km}$ dispersion compensated transmission link, and it was shown that error-free operation at the expense of small penalties is possible.

\section{Acknowledgments}

This work is supported by the European Commission project ICT-BOOM withn the 7th framework program (FP7), information and communications technology.

\section{References}

1 D.J. Blumenthal, ECOC'04, 830-833 (2004).

2 S. Zhang et al., Opt. Lett., 35, 37-39 (2005).

3 M. Jinno, Opt. Lett., 18, 1409 (1993). 